\title{
LE RÉV. PÈRE VAAST VAN BULCK, S.J.
}

LE R. P. VAAST VAN BULCK, qui fut membre du Conseil exécutif de l'Institut International Africain de 1957 à 1965 , est décédé à Louvain le 6 juillet 1966.

Professeur extraordinaire à l'Université Catholique de Louvain et professeur à l'Université pontificale grégorienne de Rome, le P. Van Bulck naquit à Berchem (Anvers) le 24 septembre 1903. Au terme de ses études secondaires, il entra à la Compagnie de Jésus où il termina en 1928 sa licence en philosophie. Après un séjour d'un an à la Sorbonne où il conquit les diplômes des Instituts de linguistique, d'ethnologie et de phonétique, il reçut au jury central à Bruxelles celui de docteur en philologie classique. En I93I, après un séjour de deux ans à Vienne où il fut formé par Schmidt et Koppers à l'ethnologie et à la linguistique africaines, il y obtint le doctorat en philosophie avec une thèse intitulée 'Beiträge zur Methodik der Völkerkunde'. Il voyagea ensuite en Afrique occidentale et centrale de I93 I à 1933 et revint en Belgique pour y achever ses études théologiques et s'y faire ordonner prêtre en 1936 .

En 1938, l'Université pontificale grégorienne de Rome lui confia la chaire d'histoire des religions, d'anthropologie, d'ethnographie et d'ethnologie. Il retourna en Afrique en 1939 pour y étudier le problème de l'unification des langues des Bantous méridionaux. Surpris par la guerre, il fut envoyé par ses supérieurs au Kwango où il résida jusqu' en I945 en poursuivant ses recherches ethnologiques et linguistiques, spécialement chez les Yaka. Il fut ensuite attaché pendant deux ans au petit séminaire de Lemfu. Après avoir exercé pendant quelques mois, à titre intérimaire, la direction du Centre Universitaire Congolais Lovanium à Kisantu, il rentra en Europe en 1947 pour y reprendre son enseignement à Rome.

Après un court séjour à la London School of Oriental and African Studies, il fut envoyé en Afrique, sous les auspices de l'Institut International Africain, pour prendre part à une expédition internationale chargée d'étudier les langues et dialectes parlés le long de la frontière séparant la zone dite soudanaise de la zone dite bantoue et allant du Cameroun jusqu'au Nil. Le P. Van Bulck fit partie de l'expédition qui, de septembre 1950 à janvier $195 \mathrm{I}$, explora la région située entre Libenge, au nord-ouest du Congo belge, et Juba sur le Nil. Il prolongea son séjour en Afrique de quelques mois en poursuivant des recherches linguistiques dans la région du Tchad.

En octobre 195 I, le R. P. Van Bulck fut nommé président de l'Institut Africain de l'Université de Louvain où il enseigna l'ethnologie et la linguistique africaines jusqu'à son éméritat en 1965 , tout en poursuivant de front son enseignement à l'Université grégorienne.

Ethnologue, linguiste, historien, et missiologue, le R. P. Van Bulck fut un travailleur indefatigable et doué d'une érudition extraordinaire. Ses nombreuses publications et sa participation active aux congrès internationaux lui valurent une notoriété certaine dans tous les milieux scientifiques africanistes. Aussi devint-il membre de l'Académie Royale des Sciences d'Outre-Mer à Bruxelles, de la Société de Linguistique de Paris, de l'Anthropos-Institut de Vienne et Fribourg, de la Nederlandse Vereniging voor Culturele Anthropologie à Amsterdam et, enfin, membre du Conseil exécutif de l'Institut international africain. 
Le P. Van Bulck était un homme qui cachait une humilité exemplaire dans une nature passionnée. Il mettait une conscience scrupuleuse à remplir les tâches qui lui étaient confiées. D'une curiosité toujours en éveil, il accumulait les notes de lecture qu'il avait hâte de mettre à profit dans ses travaux scientifiques et dans son enseignement ou qu'il récoltait simplement dans de petits cahiers en attendant de pouvoir les inclure dans de vastes publications scientifiques dont il multipliait les projets. Sa barbe blanche en broussaille cachant un visage décharné et son extrême maigreur que soulignaient des vêtements toujours trop larges témoignaient à eux seuls de l'ascèse de sa vie et l'auraient fait passer pour plus agé qu'il n'était s'il n'y avait eu le feu de son regard, la nervosité de sa démarche, et la vivacité de ses réparties. Toujours sur la brèche et ne s'accordant jamais le moindre répit, le P. Van Bulck qui n'était pas homme à se ménager la santé, fut littéralement usé par le travail. Il fut terrassé par une crise cardiaque, laissant une œuvre, inachevée certes, mais combien riche cependant sur le plan des connaissances ethnologiques et linguistiques. Le R. P. Van Bulck restera longtemps dans le souvenir de tous ceux qui savent reconnaître et respecter une vie consacrée toute entière, dans l'oubli de soi, à la recherche de la vérité.

G.M. 